\title{
Gene Variants in Predicting BCG Response to Urinary Bladder Cancer
}

\author{
Rama Devi Mittal
}

Published online: 11 February 2012

(C) Association of Clinical Biochemists of India 2012

Bladder cancer (BC) is one of the most widespread cancers distressing men and women and thus has a philosophical impact on health care. An extensive variability in the occurrence of $\mathrm{BC}$ reflects its multifactorial with polygenic etiology. Numerous risk factors including lifestyle, occupations, dietary factors, drugs, urologic pathologies, family history, and genetic variants play a significant role in the pathogenesis of $\mathrm{BC}$ [1]. $\mathrm{BC}$ is now recognized as a disease of alterations in several cellular processes. The more prevalent, less aggressive, recurrent, noninvasive tumors are characterized by constitutive activation more aggressive invasive tumors, which have a higher mortality rate, are characterized by alterations in the p53 and retinoblastoma pathways [2]. Several diagnostic tests have attempted to identify these molecular alterations in bladder tumor cells exfoliated in the urine, whereas prognostic tests have tried to identify aberrations so as to predict tumor behavior and identify therapeutic targets.

The future of BC patient management would rely on the use of molecular tests to diagnose the presence of disease, predict individual tumor behavior, and suggest potential targeted therapeutics and follow-up regime

\section{Cytokine Gene Variants and its Association with Bladder Cancer after BCG Therapy}

BCG remains the most effective intravesical treatment in non-muscle-invasive bladder cancer (NMIBC) from last

R. D. Mittal ( $\square)$

Department of Urology and Renal Transplantation,

Sanjay Gandhi Post Graduate Institute of Medical Sciences,

Raebareli Road, Lucknow 226014, Uttar Pradesh, India

e-mail: ramamittal@gmail.com; rdm_pgi@rediffmail.com three decades. Although the exact mechanisms through which BCG mediates anti-tumor immunity remain unclear, a local nonspecific immune reaction reflecting various activities of immune cells and cytokines has been proposed. Genes encoding cytokines and related molecules harbor polymorphic regions, which alter gene transcription and thereby influence inflammatory processes. Thus, specific immunomodulatory gene polymorphism associated with gene regulation and protein expression may influence genetic susceptibility and clinical outcome of a number of diseases states, such as malignancy [3].

\section{Pro- and Anti-inflammatory Cytokines Gene Variants and their Correlation with BCG Response}

Interleukin-1 (IL-1) family is a key immunoregulatory cytokine and participates in several immuno-mediated diseases. IL- $1 \alpha$ and IL- $1 \beta$ are potent pro-inflammatory cytokines, while IL-1Ra as anti-inflammatory cytokine competes with IL- $1 \alpha$ and IL- $1 \beta$ in binding to IL- 1 receptor and down-regulates the immune response and inflammation. [4]. IL-1 in association with other pro-inflammatory cytokines $(\mathrm{TNF} \alpha)$ provides survival signals to malignant cells via activating $\mathrm{NF}-\kappa \mathrm{B}$ transcription factor [5] We investigated the association of IL-1 family gene variants with increased risk of developing bladder cancer and reported the significant association of IL-1Ra with BC [6]. Based on our preliminary report, it may be very informative to study the significance of BCG therapy response with IL-1 gene variants in different populations. TNF- $\alpha$ is one of the major mediators of inflammation and has been linked to all steps involved in tumorigenesis, including cellular transformation, promotion, survival, proliferation, invasion, angiogenesis, and metastasis [7].Tumor cells secrete 
their own TNF- $\alpha$ in anticrime manner which further enhance the expression of other growth factors such as transforming growth factor-alpha (TGF- $\alpha$ ) and epidermal growth factor receptor (EGFR), both of which mediate proliferation [8]. In an attempt to monitor the immunological effects of BCG therapy, Bohle et al. [9] analyzed the urine of patients following the sixth intravesical instillation. Within $24 \mathrm{~h}$ following the instillation, all patients exhibited a strong increase in urinary TNF secretion, which was significantly different from the control group. We already reported the significant association of TNF gene variants in different diseases $[10,11]$, but there is no report with respect to $\mathrm{BC}$. On the basis of the previous reports which indicated positive correlation of TNF secretion and BCG therapy, further studies may be warranted in different cohorts with respect to TNF gene variants and BCG therapy which may give better insight for understanding its mechanism.

TGF- $\beta$ is a potent inhibitor of proliferation in epithelial cells and acts as a tumor suppressor [12]. Additionally, continuous expression of TGF- $\beta$ by cancerous cells helps cancer to progress further [13]. Sharif-Afshar et al. [14] suggested a role of stromal TGF- $\beta$ signaling with estrogens and androgens in bladder fibrosis. In a study, urine examination by immunoelectron microscopic and immunocytochemical techniques revealed a significant boost in the percentage of $\mathrm{BC}$ cases expressing TGF- $\beta$ protein. However, [15] in a case-control study reported no association of TGF- $\beta+869$ polymorphism with BC susceptibility. Interestingly, TGF- $\beta$ TT genotype carriers were observed to be associated with reduced (hazard ratio (HR) 0.37) and enhanced (HR 2.24) risk of recurrence after BC immunotherapy, respectively [16].

Interleukin-4 is a key cytokine that induces the activation and differentiation of $\mathrm{B}$ cells, and the development of the Th2 subset of lymphocytes. Th2 cytokines such as IL-4, IL-6, and IL-10 primarily support antibody production, and many studies have confirmed that patients with cancer have high levels of such cytokines in their serum [17]. Interleukin-4 also inhibits macrophage activation and might be involved in cancer formation. Many studies have confirmed that cancer patients have high levels of Th2 cytokines such as IL-10 and IL-6 including IL-4 [18]. There is also a transient increase in IL-4 levels in the urine of BC patients within $24 \mathrm{~h}$ after instillation of BCG [19]. But on the other hand IL-4 level was significantly reduced in $\mathrm{BC}$ patients after instillation of combination immunotherapy [20]. Studies from our group reported no association of BC risk and BCG therapy with polymorphism in IL-4 intron3 [21]. However, more studies in larger cohort need to be done to explore the exact mechanism and correlation of IL-4 gene with BCG therapy in $\mathrm{BC}$ patients.
In the genitourinary system, IL-6 may transform urothelial cells and provide selective growth advantage to urothelial cancerous cells [22]. In vitro study demonstrated that urothelial malignant cells secrete a large amount of IL-6 compared to normal urothelium, and the addition of anti-IL-6 antibody and antisense oligonucleotide significantly reduced cancerous cell growth [23]. Simultaneously, significant elevated levels of IL-6 were reported in urine of UBC patients [24]. Cardillo et al. [25] reported by immuno-histochemistry expression that high-grade and muscle-invasive bladder tumors contained significantly higher levels of IL-6 proteins. The possible relationship between BCG internalization and IL-6 up-regulation was studied using T24 bladder cancer cell line who observed up regulation of IL- 6 synthesis to be inhibited by $\alpha$ BCG of IL6 production due to BCG internalization by poorly differentiated bladder carcinoma cells may be part of the mode of action of intravesical BCG therapy. BCG induces cAMP production and may regulate interleukin- 6 expression partially via a cAMP-dependent pathway in human bladder cancer cells [26]. Since $174 \mathrm{G}>\mathrm{C}$ polymorphism of IL-6 is functionally active, many groups studied its association with BC including ours [21]; [27].

IL-10 is an important immunoregulatory cytokine with pleiotropic effects. It is produced by many activated immune cell types, including T helper (Th2) cells, B cells, macrophages, monocytes, and keratinocytes [28]. It limits inflammation and modulates immune response by inhibiting the production of various pro-inflammatory and Th1 cytokines and blocking the expression of MHC class II and co-stimulatory molecules on the surface of antigen-presenting cells [29-30]. Inhibitory role of IL-10 in BCGinduced macrophage cytotoxicity suggests that blockage of IL-10 may potentially enhance the effect of BCG in the treatment for bladder cancer patients [31]. We suggested that the low-producing genotypes of IL-10 (C-819T and G-1082A) polymorphisms are associated with increased $\mathrm{BC}$ risk. Individuals with (C-819T) TT genotype and T carriers, however, showed a protective association with high-risk tumors [16]. IFN- $\gamma$ is a pro-inflammatory cytokine that promotes allo-immune responses by enhancing lymphocyte function, stimulating expression of adhesion molecules, and up-regulating MHC class-I and MHC classII expression IFN- $\gamma$ is secreted by subsets of activated $\mathrm{CD} 4+$ and $\mathrm{CD} 8+\mathrm{T}$ cells as well as activated natural killer (NK) cells and exerts its enormous variety of effects via a specific IFN- $\gamma$ receptor. IFN- $\gamma$ is a potent BCG enhancer that polarizes the BCG-induced immune response toward the cellular immune pathway by promoting Th1 cytokine expression and reducing Th2 cytokine expression [32]. [33] showed anti-proliferative activity of IFN gamma in SBC. Interferon-gamma has also shown inhibitory effect on the growth of three human BC cell lines, RT4, RT112, and 
MGH-U1, representing tumor grades 1, 2 and 3, respectively. In our study, increased risk was observed with the IFN-G +874 A allele (OR 1.78). IFN- $\gamma+874$ A carriers were associated with enhanced (HR 2.24) risk of recurrence after BCG immunotherapy, respectively [16]. In summary, all the cytokines are key immune mediators and play an important role in inflammation-induced cancers including BC. Cytokine gene variants have been shown to account for differences in inter-individual variability for various cancers including $\mathrm{BC}$. The collective influence of several cytokines could influence immune responses as complex as those underlying cancer initiation and modified response to therapeutics. Ethnic differences in cytokine gene variants correlate with varied population-based risk of BC. With the recent knowledge on cytokine gene variants, the potential value of its effect on recurrence/progression of $\mathrm{BC}$ could be implicated for the management of longterm recurrence-free survival.

\section{Mechanism in BCG Immunotherapy in Bladder Cancer}

BCG has now been well established to delay the recurrence of high-risk (high-grade, large, multiple, and recurrent tumors) non-muscle-invasive bladder tumors. Though the precise sequential events in the immunological response elicited after BCG instillation are ambiguous yet, the possible sequence may be as follows. After Instillation, BCG get internalized to urothelial cells via Fibronectin protein This results in the induction of chemokines such as IL-8 and inflammatory cytokines and up-regulation of adhesion molecule expression [34]. Simultaneously, a plethora of cytokines including granulocyte-macrophage colony-stimulating factor, TNF- $\alpha$ IFN- $\gamma$ IL-6, IL-2, IL-5, and IL-12 have been detected in the urine of patients after the BCG immunotherapy [20]. These secreted cytokines suggest that BCG predominantly produces $\mathrm{T}$ helper type 1 (Th1) immune response in patients and ultimately activates cytotoxic $\mathrm{T}$ cells (CD8+), macrophages, and natural killer cells (NK cells) against tumor cells [34]; Hence, it is clear that BCG basically strengthens body's immune response via enhancing Th1 cytokine production (IFN- $\gamma$ TNF- $\alpha$ etc.), to combat recurrence of tumor. Another emerging therapy in $\mathrm{BC}$ with great clinical potential is retinoic acid treatment. This again utilizes cytokines like VEGF and TGF- $\beta$ to reduce risk of recurrence. The reduced rate of recurrence and increased survival time after retinoic acid treatment has been reported [35]. Thus, cytokines are crucial players in the mechanism of BCG action, and inter-individual variability in these cytokine expressions may modify clinical outcome of BCG treatment.

These rationales urged researchers to explore the association of cytokine gene polymorphisms with clinical outcome after BCG immunotherapy in different ethnic regions. Since, these polymorphisms ultimately affect protein activity/levels, many researchers tried to correlate cytokine levels in urine or serum with chance of recurrence and progression. Numerous studies have confirmed the presence of a large variety of cytokines in urine and serum after intravesical BCG instillation, including IL-1, IL-2, IL-6, IL-8, IL-10, IL-12, TNF- $\alpha$ and IFN- $\gamma[34,36]$. Most of these cytokines are involved in initiation and strengthening Th1 of immune response. As discussed above, these cytokines strengthen cell-mediated immunity against tumor. The drastic increase in urinary levels of IFN- $\gamma$ and other Th1 cytokines (IL-2 and TNF) is associated with longer recurrence-free survival, whereas higher levels of $\mathrm{Th} 2$ cytokines (IL-10) are associated with BCG failure [36]. Therefore, polymorphisms affecting the Th1/Th2 balance may determine the efficacy of BCG immunotherapy.

The same therapeutic index of medication always causes considerable heterogeneity in efficacy and toxicity across human population. This heterogeneity can even lead to unpredictable life-threatening and lethal adverse effects in a group of patients. Though the BCG immunotherapy has gained a strong believe as an effective treatment option for high-risk superficial UBC, similar observation was reported with BCG treatment, where $30-35 \%$ of clinically classified similar patients at high risk of recurrence were not responding to the treatment and demonstrated reduced recurrence free survival. This unexplained BCG failure results in more economic burden, more follow-ups, and uneasy life. The inter-individual variability in drug response cannot satisfactorily be explained by patients' age, life style, or co-medication and compliance of patients. The solution is probably doomed to be individual differences in their genomics. The identification of these genetic factors perhaps in cytokine gene may explain part of 30-35\% failure of BCG immunotherapy for high-risk tumors and help to optimize its efficacy.

\section{Cytokine Gene Variants along with other Novel Approaches in BC}

The limitations of BCG immunotherapy have led to the exploration of the immunostimulatory potential of bacterial components or genetically modified bacteria. Therefore, alternative treatment strategies using nonviable sub fractions of mycobacterium have been explored. Although few promising results were observed, for example, cell wall extracts [37], others show that the viability of BCG (and of active intracellular infection) is of prime importance for the efficiency of this immunotherapy. Therefore, nonviable alternatives to BCG would most likely not be equally effective until adequate delivery systems have been 
developed and proper retention of the material in the bladder is ensured. Since cytokines are key mediators of anti-tumor activity of BCG and may also eradicate tumor cells directly, current studies focus on cytokine and BCG combination to achieve highest efficacy and least side effects. IFN- $\gamma$ and IL-12 seem to be the promising candidates and they have already shown some initial success in patients with bladder cancer.

\section{Conclusion}

Many details of the molecular and cellular mechanisms involved in BCG immunotherapy of BC have been discovered in the past few decades. However, information on a potential role for cytokine gene variants as an effecter mechanism is limited. Since BC is a multifactorial and polygenic disorder, there is need to study the exact risk factors and the complex interactions between multiple genes and environmental exposures through epidemiological studies. Pathway-based genotyping of more SNPs in inflammation genes and haplotype analyses are warranted to confirm and extend our findings. Since there is a scarcity of data, additional epidemiological data from different populations and centers are desirable. Simultaneously, we attempted to limit the confounding effect of ethnicity by restricting our analysis to Indian subjects; however, the place of origin of ancestors among these participants could possibly affect the frequencies of certain genotypes and cause spurious associations.

Cytokine therapy and BCG immunotherapy. Information could provide urologists with the ability to optimize required dose of immunoregulatory cytokines. The studies analyzing association of these SNPs and genetic prowling of patients may help them to decide optimum dose and proper regime of BCG immunotherapy. The inclusion of genetic information in active surveillance protocol may reduce the amount of surgical intervention the patients might have to undergo throughout their lives. In this way, personalized treatment can be tailored according to genetic makeup of a patient and may improve the quality of life and decrease economic burden as well. Identification of a broad genetic determinant for $\mathrm{BC}$ initiation and outcome after BCG immunotherapy offers the opportunity not only to tailor treatment regime but also to explore underlying mechanism and to identify novel therapeutic targets.

\section{References}

1. Mitra AP, Cote RJ. Molecular pathogenesis and diagnostics of bladder cancer. Annu Rev Pathol. 2009;4:251-85.
2. ReznikoV CA, Sarkar S, Julicher KP, et al. Genetic alterations and biological pathways in human bladder cancer pathogenesis. Urol Oncol. 2000;5:191-203.

3. Bidwell J, Keen L, Gallagher G, et al. Cytokine gene polymorphism in human disease: on-line databases, supplement 1 . Genes Immunol. 2001;2:61-70.

4. Arend WP, Malyak M, Guthridge CJ, Gabay C. Interleukin-1 receptor antagonist: role in biology. Annu Rev Immunol. 1998; 16:27-55.

5. Balkwill F, Charles KA, Mantovani A. Smoldering and polarized inflammation in the initiation and promotion of malignant disease. Cancer Cell. 2005;7:211-7.

6. Bid HK, Manchanda PK, Mittal RD. Association of interleukin$1 \mathrm{Ra}$ gene polymorphism in patients with bladder cancer: case control study from North India. Urology. 2006;67:1099-104.

7. Sethi G, Sung B, Aggarwal B. TNF: a master switch for inflammation to cancer. Front Biosci. 2008;13:5094-107.

8. Schmiegel W, Roeder C, Schmielau J, Rodeck U, Kaltho VH. Tumor necrosis factor alpha induces the expression of transforming growth factor alpha and the epidermal growth factor receptor. Proc Natl Acad Sci USA. 1993;90:863-7.

9. Bohle A, Nowc C, Ulmer AJ, et al. Detection of urinary TNF, IL1, and IL 2 after local BCG immunotherapy for bladder carcinoma. Cytokine. 1990;2:175-81.

10. Singh P, Francis A, Bid HK, et al. Role of ethnic variations inTNF-alpha and TNF-beta polymorphisms and risk of breast cancer in India. Breast Cancer Res Treat. 2011;126:739-47.

11. Mittal RD, Manchanda PK, Bid HK, Ghoshal UC. Analysis of polymorphisms of tumor necrosis factor-alpha and polymorphic xenobiotic metabolizing enzymes in inflammatory bowel disease:study from northern India. J Gastroenterol Hepato. 2007;22: $920-4$.

12. Siegel PM, Massague J. Cytostatic and apoptotic actions of TGFbeta in homeostasis and cancer. Nat Rev Cancer. 2003;3:807-21.

13. Davis BN, Hilyard AC, Lagna G, Hata A. SMAD proteinscontrol DROSHA-mediated microRNA maturation. Nature. 2008;454: 56-61.

14. Sharif-Afshar AR, Donohoe JM, Pope JCT, Adams MC, Brock JW III, Bhowmick NA. Stromal hyperplasia in male bladders upon loss of transforming growth factor-beta signaling in fibroblasts. J Urol. 2005;174:1704-7.

15. Castillejo A, Rothman N, Murta-Nascimento C, et al. TGFB1 and TGFBR1 polymorphic variants in relationship to bladder cancer risk and prognosis. Int J Cancer. 2009;124:608-13.

16. Ahirwar DK, Agrahari A, Mandhani A, Mittal RD. Cytokine gene polymorphisms are associated with risk of urinary bladder cancer and recurrence after BCG immunotherapy. Biomarkers. 2009;14: 213-8.

17. Groah SL, Weitzenkamp DA, Lammertse DP, Whiteneck GG, Lezotte DC, Hamman RF. Excess risk of bladder cancer in spinal cord injury: evidence for an association between indwelling catheter use and bladder cancer. Arch Phys Med Rehabil. 2002; 83:346-51.

18. Chandler SW, Rassekh $\mathrm{CH}$, Rodman SM, Ducatman BS. Immunohistochemical localization of interleukin-10 in human oral and pharyngeal carcinoma. Laryngoscope. 2002;112:808-15.

19. Sander B, Damm O, Gustafsson B, Andersson U, Hakansson L. Localization of IL-1, IL-2, IL-4, IL-8 and TNF in superficial bladder tumors treated with intravesical Bacillus Calmette-Guerin. J Urol. 1996;156:536-54.

20. Agarwal A, Agrawal U, Verma S, Mohanty NK, Saxena S. Serum Th1 and Th2 cytokine balance in patients of superficial transitional cell carcinoma of bladder pre- and post-intravesical combination immunotherapy. Immunopharmacol Immunotoxicol. 2003;32: 348-56. 
21. Ahirwar D, Kesarwani P, Manchanda PK, Mandhani A, Mittal RD. Anti- and proinflammatory cytokine gene polymorphism and genetic predisposition: association with smoking, tumor stage and grade, and Bacillus Calmette-Guerin immunotherapy in bladder cancer. Cancer Genet Cytogenet. 2008;184:1-8.

22. Okamoto M, Hattori K, Oyasu R. Interleukin-6 functions as an autocrine growth factor in human bladder carcinoma cell lines in vitro. Int J Cancer. 1997;72:149-54.

23. Okamoto M, Kawamata H, Kawai K, Oyasu R. Enhancement of transformation in vitro of a nontumorigenic rat urothelial cell line by interleukin 6. Cancer Res. 1995;55:4581-5.

24. Seguchi T, Yokokawa K, Sugao H, Nakano E, Sonoda T, Okuyama A. Interleukin-6 activity in urine and serum in patients with bladder carcinoma. J Urol. 1992;148:791-4.

25. Cardillo MR, Sale P, Di Silverio F. Heat shock protein-90, IL-6 and IL-10 in bladder cancer. Anticancer Res. 2000;20:4579-83.

26. Zhang Y, Mahendran R, Yap LL, Esuvaranathan K, Khoo HE. The signalling pathway for BCG-induced interleukin-6 production in human bladder cancer cells. Biochem Pharmacol. 2002; 63:273-82.

27. Leibovici D, Grossman HB, Dinney CP, et al. Polymorphisms in inflammation genes and bladder cancer: from initiation to recurrence, progression, and survival. J Clin Oncol. 2005;23: 5746-56.

28. Goldman M, Velu T. Interleukin-10 and its implications for immunopathology. Adv Nephrol Necker Hosp. 1995;2479-90

29. Moore KW, de Waal Malefyt R, Coffman RL, O'Garra A. Interleukin-10 and the interleukin-10 receptor. Annu Rev Immunol. 2001;19:683-765.
30. Petit-Bertron AF, Pedron T, Gross U, et al. Adherence modifies the regulation of gene expression induced by interleukin-10. Cytokine. 2005;29:1-12.

31. Luo Y, Han R, Evanoff DP, Chen X. Interleukin-10 inhibits Mycobacterium bovis Bacillus Calmette-Guerin (BCG)-induced macrophage cytotoxicity against bladder cancer cells. Clin Exp Immunol. 2010;160:359-68.

32. Luo Y, Chen X, Downs TM, DeWolf WC, O'Donnell MA. IFNalpha2B enhances Th1 cytokine responses in bladder cancer patients receiving Mycobacterium bovis Bacillus CalmetteGuerinn immunotherapy. J Immunol. 1999;162:2399-405.

33. Stavropoulos NE, Ioachim E, Pappa L, Hastazeris K, Agnantis NJ. Antiproliferative activity of interferon gamma in superficial bladder cancer. Anticancer Res. 1999;19:4529-33.

34. Kitamura H, Tsukamoto T. Immunotherapy for urothelial carcinoma: current status and perspectives. Cancers. 2011;3:3055-72.

35. Hameed DA, El-Metwally TH. The effectiveness of retinoic acid treatment in bladder cancer: impact on recurrence, survival and TGFalpha and VEGF as end-point biomarkers. Cancer Biol Ther. 2008;7:92-100.

36. Shintani Y, Sawada Y, Inagaki T, Kohjimoto Y, Uekado Y, Shinka T. Intravesical instillation therapy with Bacillus Calmette-Guerin for superficial bladder cancer: study of the mechanism of Bacillus Calmette-Guerin immunotherapy. Int J Urol. 2007;14:140-6.

37. Chin JL, Kadhim SA, Batislam E, et al. Mycobacterium cell wall: an alternative to intravesical Bacillus Calmette Guerin (BCG) therapy in orthotopic murine bladder cancer. J Urol. 1996;156: 1189-93. 